\title{
La resolución de retos energéticos y medioambientales mediante la nanotecnología: la experiencia de colaboración entre España y Japón'
}

\author{
Estrella Fernández* y Pedro A. Serena**
}

RESUMEN: Este artículo muestra el contexto, el diseño, el desarrollo y los resultados de una actuación conjunta en materia de política cientificotecnológica entre los gobiernos de España y Japón con el fin de promover la utilización de la nanotecnología para la resolución de problemas energéticos y medioambientales, mediante la financiación de proyectos conjuntos de investigación y de talleres científicos de alto nivel. El artículo revisa los principales resultados alcanzados a través de varios indicadores que demuestran el éxito de estos proyectos desarrollados en diferentes temas de impacto en energía fotovoltaica, nuevos materiales y dispositivos para generación y almacenamiento de energía, pilas de combustible, almacenamiento de hidrógeno, etc. Además, las actividades desarrolladas a lo largo de la actuación tienen efectos no sólo cuantitativos sino también intangibles, a través del intercambio de procedimientos y esquemas de gestión cientificotécnica y el establecimiento de redes de contactos e interacciones sobre los que plantear futuras actuaciones.

PALABRAS CLAVE: Política científica, colaboración internacional, nanotecnología, energía, medioambiente, sostenibilidad.

Recibido: 7 de agosto de 2015. Aceptado: 30 de octubre de 2015.

1 Se agradece al Ministerio de Economía y Competitividad la financiación del Proyecto "Fomento de la cooperación con Rusia, Australia y Asia en el Área de la nanotecnologías (NANORAA)" (ACI2010-1134) a través del Subprograma de Fomento de la Cooperación Científica Internacional, dirigido a impulsar la participación de instituciones de I+D e investigadores españoles en la esfera internacional, a promover el liderazgo de investigadores españoles en proyectos de programas internacionales, a fomentar la cooperación en ciencia y tecnología prevista en el marco de acuerdos bilaterales firmados con ciertos países, y a otras actuaciones de cooperación científica.

Se agradece a todo el personal del Ministerio de Ciencia e Innovación (MICINN) y de la Secretaría de Estado de Investigación, Desarrollo e Innovación del Ministerio de Economía y Competitividad (MINECO) que ha estado involucrado en la gestión de este programa internacional entre MICINN/MINECO y JST. Se extiende este agradecimiento al personal de la agencia JST involucrado en el desarrollo del programa, al de la Embajada de España en Tokio, y al de la Embajada de Japón en Madrid, por su apoyo en la organización de los eventos. Finalmente, agradecemos el esfuerzo de todos los investigadores participantes en las convocatorias de diferentes formas, bien presentando y ejecutando los proyectos, bien evaluando las propuestas o realizando el posterior seguimiento de los proyectos aprobados.

* Subdirección General de Proyectos Internacionales, Dirección General de Investigación Científica y Técnica, Ministerio de Economía y Competitividad (MINECO). c/ Albacete 5, E-28071-Madrid, España.

** Instituto de Ciencia de Materiales de Madrid (ICMM), Consejo Superior de Investigaciones Científicas (CSIC). c/ Sor Juana Inés de la Cruz 3, Campus de Cantoblanco, E-28049-Madrid, España. Correspondencia: (pedro.serena@icmm.csic.es); tel.: +34 913348999. 
ABSTRACT: This paper offers a review of the context, design, development and results of a joint action on science and technology policy between the governments of Spain and Japan aimed to promote the use of nanotechnology for solving energy and environmental problems, through the funding of research projects and the organization of high-level scientific workshops. The paper analyses the main outcomes using several indicators that show the impact and success of such joint action that has promoted the development of key areas such as photovoltaic energy, new materials and devices for energy generation and storage, fuel cells or hydrogen storage. Furthermore, the effects of carried out actions are not only quantitative since, as result of this joint initiative, an intensive exchange of scientific and technical management procedures and schemes has been achieved. In addition, the establishment of scientific networks and solid interactions will be very useful to planning future actions.

KEYWORDS: scientific policy, international collaboration, nanotechnology, energy, environment, sustainability.

\section{Introducción}

España fue uno de los primeros países europeos en establecer relaciones diplomáticas con Japón, hecho acontecido en el siglo XVI. Sin embargo, las relaciones de índole comercial se remontan a varios siglos a través de las importaciones de seda y especias orientales tan apreciadas en toda Europa (Noya, 2004). La conexión entre España y Japón se realizó de múltiples maneras: a través de la Compañía de Jesús, en su intento de llevar el cristianismo a los países de Extremo Oriente, mediante el famoso Galeón de Manila, con exportaciones japonesas que llegaban a España a través de México, o mediante el envío de misiones diplomáticas, como ocurrió con la "Embajada a Europa de la Era Keicho", entre 1613 y 1620, encabezada por el samurái Hasekura Tsunenaga. Con el establecimiento de las políticas de aislamiento de Japón durante la era Edo o periodo Tokugawa, entre 1603 y 1868, y la lenta pérdida de influencia de España en la geopolítica mundial a partir del siglo XVIII, las relaciones de los dos países fueron debilitándose. El primer "Tratado de amistad, comercio y navegación” entre España y Japón se firmó en 1868, año de inicio de la Era Meiji, y desde entonces las relaciones diplomáticas y comerciales se han ido consolidando, pasando por diferentes etapas. Sin embargo, en el imaginario colectivo de los habitantes de cada país siempre se ha sentido un gran respeto por la otra nación, percibiéndose entre sí como imperios lejanos, de fuertes y exóticas culturas, que han jugado un gran papel en la historia (Noya, 2004).

A raíz de la emergencia de Japón como potencia económica global tras la Segunda Guerra Mundial y el progresivo aumento de la presencia de España en la economía mundial, las relaciones económicas de ambos países se fueron consolidando, favoreciendo la aproximación de ambas sociedades. No debemos olvidar que la irrupción económica, en cadena, de Japón, Taiwán, Corea del Sur, y China en los últimos 50 años ha ido desplazando el centro de gravedad de la economía mundial hacia el continente asiático. En estos momentos cerca de 250 empresas niponas operan en España, mientras unas 30 
españolas lo hacen en territorio japonés. Por otro lado, cerca de 400,000 japoneses visitan cada año España, poniendo de manifiesto su interés por la sociedad y la cultura españolas. Esta aproximación se ha hecho más patente en los últimos 20 años, a través de la puesta en marcha de iniciativas para lograr el acercamiento de ambos países en el ámbito de la sociedad civil. Un ejemplo lo tenemos en el Foro España Japón, organizado por la Fundación Consejo España Japón (Foro España Japón, 2015) que se reúne anualmente de forma alterna en cada uno de los dos países para fomentar las redes de contactos entre profesionales, empresas e instituciones niponas y españolas. El primer Foro España Japón se celebró en Madrid en 1997 y desde entonces ha tenido lugar en otras quince ocasiones. La última reunión del Foro España Japón tuvo lugar en noviembre de 2014 en Santander (España) dirigido a establecer contactos en relación con el tema emergente de "Ciudades Inteligentes”, que cuenta con interesantes ejemplos en Japón y España. En dicho Foro se presentó un informe realizado por el Real Instituto Elcano, centrado en las relaciones entre España y Japón en los ámbitos económico-empresarial, científico-tecnológico, educativo, cultural y turístico (Esteban, 2014).

El incremento de las relaciones comerciales, culturales y civiles entre España y Japón, experimentado en el último medio siglo, no tuvo inicialmente un equivalente en el auge de las relaciones cientificotecnológicas, sobre todo por el escaso peso de la ciencia y la tecnología españolas, hasta la década de 1990, en comparación con las japonesas. Esto ha hecho que la ciencia española, con un rápido crecimiento en los últimos 30 años, no haya tenido mucha penetración en Japón o en el resto de los países asiáticos. Las relaciones científicas españolas, tanto a nivel gubernamental como las establecidas a otros niveles desde universidades u organismos públicos de investigación son más intensas con los países de la Europa occidental, los países iberoamericanos, y EEUU, mientras que las conexiones con Asia, los países del este de Europa, África y Oceanía han sido mucho más débiles. A nivel de grupos de investigación la colaboración científica entre España y Japón se ha realizado a través de contactos en congresos o mediante el uso de grandes instalaciones, como el sincrotrón ubicado en Tsukuba, auténtica ciudad de la ciencia japonesa, en la que se ubican grandes centros de investigación como el Instituto Nacional de Ciencia de Materiales (NIMS) (NIMS, 2015) o el Instituto Nacional Ciencia y Tecnologías Industriales Avanzadas (AIST) (AIST, 2015).

Para fortalecer estas débiles relaciones institucionales con Japón en el ámbito científico-técnico en los últimos años se han firmado diferentes acuerdos. En diciembre de 2008, se firmó un acuerdo entre la Organización para el Desarrollo de Nuevas Energías y Tecnología Industrial de Japón (NEDO) (NEDO, 2015) y el Centro para el Desarrollo Tecnológico Industrial (CDTI) (CDTI, 2015), entonces dependiente del Ministerio de Ciencia e Innovación (MICINN), cuyas competencias han pasado al actual Ministerio de Economía y Competitividad (MINECO) (MINECO, 2015). Dicho acuerdo 
puso en marcha el Programa Bilateral de Cooperación Tecnológica Japan and Spain Innovation Program (JSIP), primer convenio de esta naturaleza que la agencia japonesa NEDO firmó con otro país, con el que se iniciaron proyectos en los que participan empresas españolas como Acciona o Endesa, o las japonesas Mitstubishi Motors o Hitachi, entre otras. Por otro lado, en febrero de 2009, se firmó el acuerdo de cooperación científica y académica entre el Instituto Nacional de Ciencia de Fusión de Japón (NIFS) (NIFS, 2015) y el Centro de Investigaciones Energéticas, Medioambientales y Tecnológicas (CIEMAT) (CIEMAT, 2015). En el marco de este acuerdo el CIEMAT ha diseñado el primer elemento del proyecto de fusión JT60SA instalado en el centro Naka Fusión Institute (NFI, 2015), perteneciente a la Agencia de Energía Atómica de Japón, y que forma parte del proyecto ITER (ITER, 2015), un dispositivo experimental de fusión nuclear que se está construyendo en Francia resultado de la cooperación internacional.

Esta secuencia de convenios entre España y Japón dio lugar a un acuerdo de mucho mayor calado entre ambos países para fortalecer la cooperación científica e impulsar la innovación empresarial, mediante el incremento de intercambio científico y el desarrollo tecnológico conjunto entre empresas españolas y japonesas. El acuerdo fue firmado el 1 de septiembre de 2010 entre el Ministerio de Educación, Cultura, Deportes, Ciencia y Tecnología (MEXT, 2015) de Japón y el Ministerio de Ciencia e Innovación (MICINN) de España. Dentro de este acuerdo, la biotecnología y la nanotecnología destacan como prioridades de la colaboración por la existencia en ambos países de grupos de investigación y empresas con oportunidades de liderazgo a nivel internacional. Como parte de este acuerdo, durante el año 2011, el Ministerio de Educación, Cultura, Deportes, Ciencia y Tecnología envió una delegación a España que realizó un informe completo sobre la estructura del sistema español de investigación, desarrollo e innovación en España, que ha servido para que las autoridades japonesas amplíen su conocimiento sobre el potencial español en muchos ámbitos de la investigación y la innovación.

Los anteriores ejemplos ponen de manifiesto cómo Japón y España han acelerado sus relaciones en el ámbito cientificotecnológico. Como ya se ha mencionado anteriormente, la nanotecnología ha sido un eje central en el nuevo esquema de relaciones entre los dos países, sin duda alguna porque ambas naciones son conocedoras del importante papel de la nanotecnología en el desarrollo industrial y tecnológico del siglo XXI (Delgado, 2009; Serena, 2009). La siguiente sección profundiza en el papel de la nanotecnología en las relaciones cientificotecnológicas entre España y Japón.

\section{Fraguando contactos entre España y Japón en el contexto de la nanotecnología}

En el año 2007, el Instituto Español para el Comercio Exterior (ahora convertido en la entidad ICEX España Exportación e Inversiones) (ICEX, 2015) 
y la Fundación Phantoms (Phantoms, 2015) firmaron un acuerdo que ha permitido mostrar en ferias comerciales internacionales el potencial de empresas e instituciones españolas en el emergente campo de la nanotecnología. La estrategia de ICEX para promocionar empresas de sectores emergentes ha sido un elemento decisivo para fomentar las relaciones España-Japón en el ámbito de la nanotecnología. Por su parte, la Fundación Phantoms es un referente internacional puesto que coordina la Red NanoSpain (NanoSpain, 2015), formada por más de 360 grupos de investigación, y organiza grandes eventos internacionales en el ámbito de la nanotecnología como la afamada serie de congresos Trends in Nanotechnology y la Feria Internacional ImagineNano.

Como fruto de esta colaboración entre el ICEX y la Fundación Phantoms, en el año 2008 se presentó por primera vez un Pabellón de España en la mayor feria del mundo dedicada a nanotecnología NanoTech Tokyo (Nanotech Tokyo, 2015), que se celebra anualmente en Tokio desde el año 2002. La presencia de una amplia representación de empresas, centros de investigación y universidades españolas en esta gran feria internacional no dejó indiferente a las autoridades japonesas, quienes enviaron en 2008 delegaciones a España con el fin de detectar la fortaleza de sus centros de investigación y universidades en este campo y promover una posible colaboración institucional. Durante el año 2008, también se realizó un estudio en el entonces MICINN de las relaciones existentes entre centros españoles y japoneses en el ámbito de la nanotecnología. La figura 1 muestra el esquema de las principales colaboraciones existentes antes de 2008 entre entidades japonesas y españolas en el campo de la nanotecnología. Esta figura se elaboró a partir de un estudio de las publicaciones internacionales conjuntas en temáticas relacionadas con la nanociencia y la nanotecnología firmadas simultáneamente por autores españoles y japoneses.

El fruto de estos encuentros y estudios fue la firma, el 11 de marzo de 2009, de un Memorando de Entendimiento (MoU) entre el Ministerio de Ciencia e Innovación (MICINN) y la Agencia Japonesa de Ciencia y Tecnología (JST) (JST, 2015) para la puesta en marcha de un Programa Bilateral de Cooperación Científica y Tecnológica. La agencia JST depende del Ministerio de Educación Cultura, Deporte, Ciencia y Tecnología (MEXT, 2015). Dicho acuerdo contemplaba diversos niveles de colaboración en todas las temáticas relacionadas con el desarrollo sostenible, incluyendo celebraciones de jornadas y talleres, envío de delegaciones, convocatorias conjuntas de proyectos de investigación, etc. En el marco de este acuerdo se celebró, en abril de 2009, en Tokio, el primer taller hispanojaponés sobre "Nanociencia y Nuevos Materiales" (figura 2) organizado de forma conjunta por la agencia JST y el MICINN (JST-MICINN, 2009). En el mismo, se intercambiaron experiencias en la resolución de problemas relacionados con el medioambiente y con la generación, conversión, transporte y almacenamiento de energía usando aproximaciones derivadas de la nanociencia y la nanotecnología. La 
FIGURA 1. Esquema de conexiones de mayor relevancia entre centros españoles (círculos rojos) y japoneses (cuadrados negros) con anterioridad a 2008 en el ámbito de la nanotecnología. La parte inferior de la figura contiene las siglas de los centros e institutos.
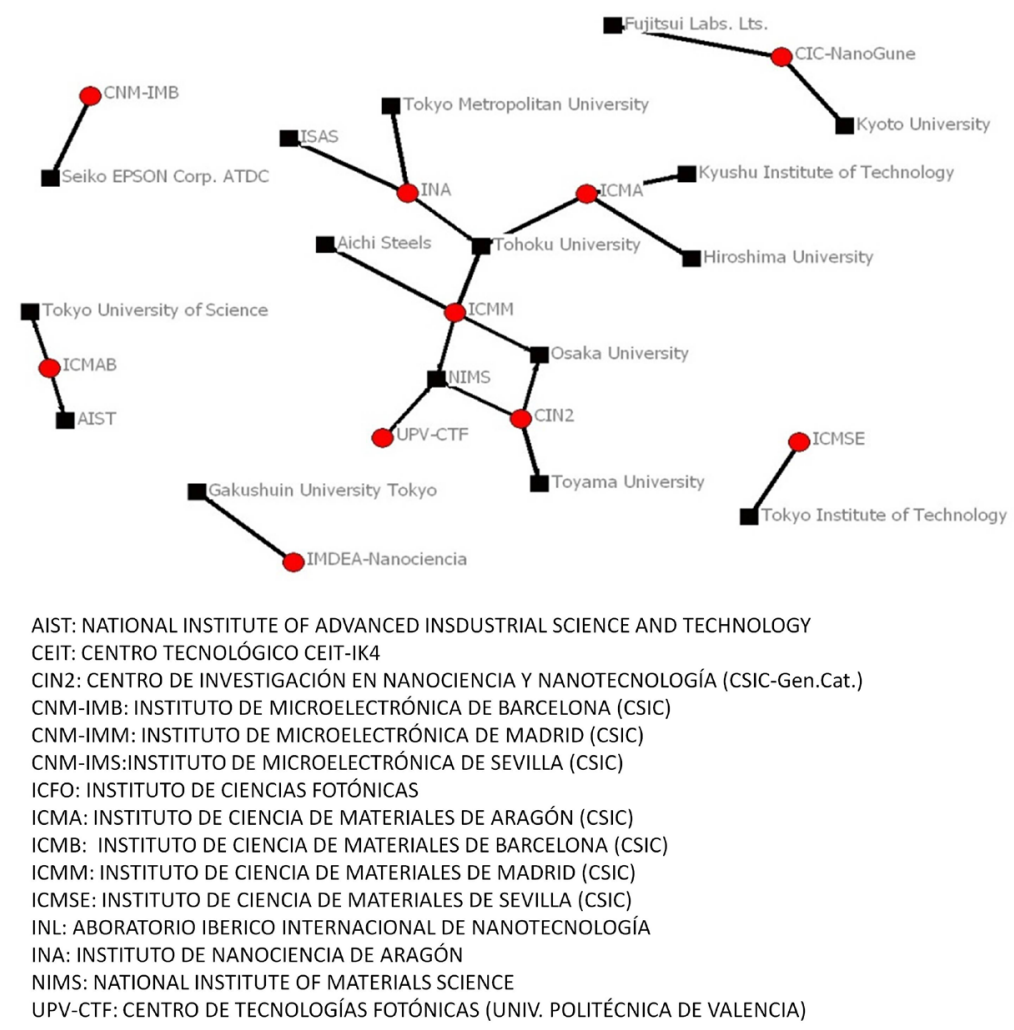

Fuente: Elaboración propia.

delegación española tuvo la oportunidad de visitar centros de investigación como NIMS, AIST y la Universidad de Tokio.

En marzo de 2010, se celebró en Barcelona el taller hispanojaponés sobre "Nanotecnología para los retos energéticos y medioambientales" organizado nuevamente por la agencia JST y el MICINN, con la colaboración de Casa Asia y del Consejo Superior de Investigaciones Científicas (CSIC) (CSIC, 2015). Este taller mostró las capacidades de ambos países y fue la antesala de una convocatoria conjunta entre JST y MICINN de proyectos de investigación que mencionaremos más adelante La reunión científica incluyó la visita al Instituto de Ciencia de Materiales de Barcelona (ICMAB), al Instituto de Microelectrónica de Barcelona (IMB) (figura 3) y al sincrotrón ALBA.

Para finalizar esta sección, se debe mencionar que en 2012 la política científica del Gobierno de España pasó a ser competencia de la Secretaría de Estado de Investigación, Desarrollo e Innovación (SEIDI) dependiente del Ministerio de Economía y Competitividad (MINECO), tras la desaparición 
Mundo Nano | Artículos | vol. 8, No. 15, julio-diciembre, 2015 | www.mundonano.unam.mx

FIGURA 2. Participantes en el taller conjunto hispanojaponés celebrado en Tokio en abril de 2009.

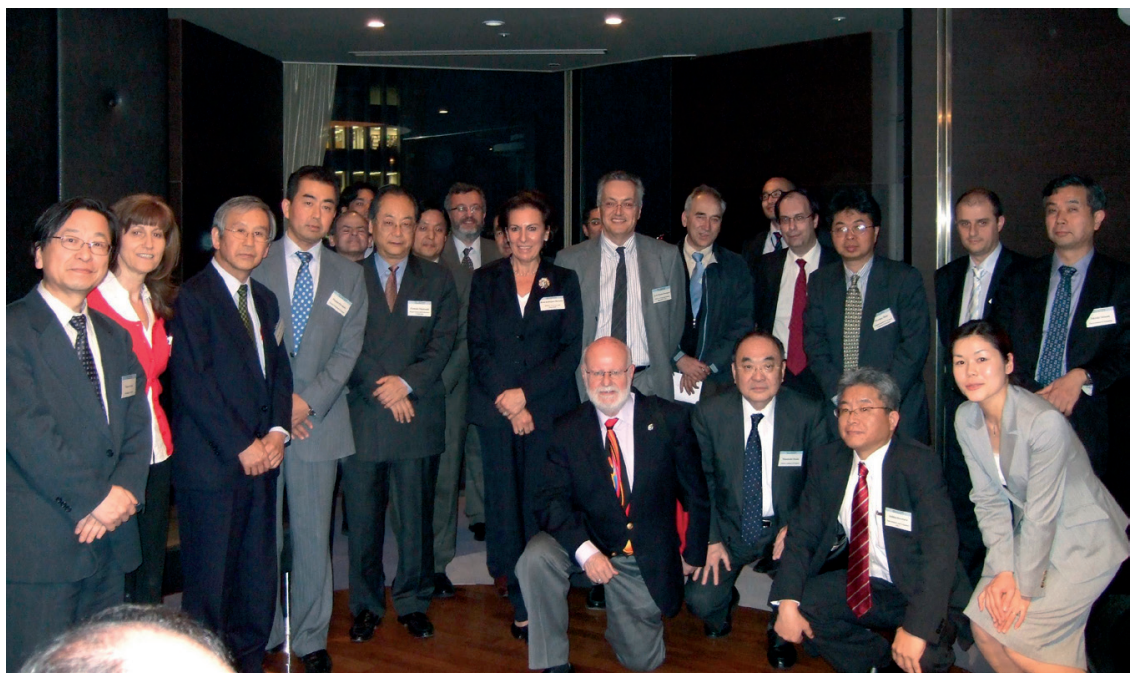

Fuente: Elaboración propia.

FIGURA 3. Participantes en el taller conjunto hispanojaponés celebrado en Barcelona en 2010 durante su visita al Instituto de Microelectrónica de Barcelona, perteneciente al Consejo Superior de Investigaciones Científicas.

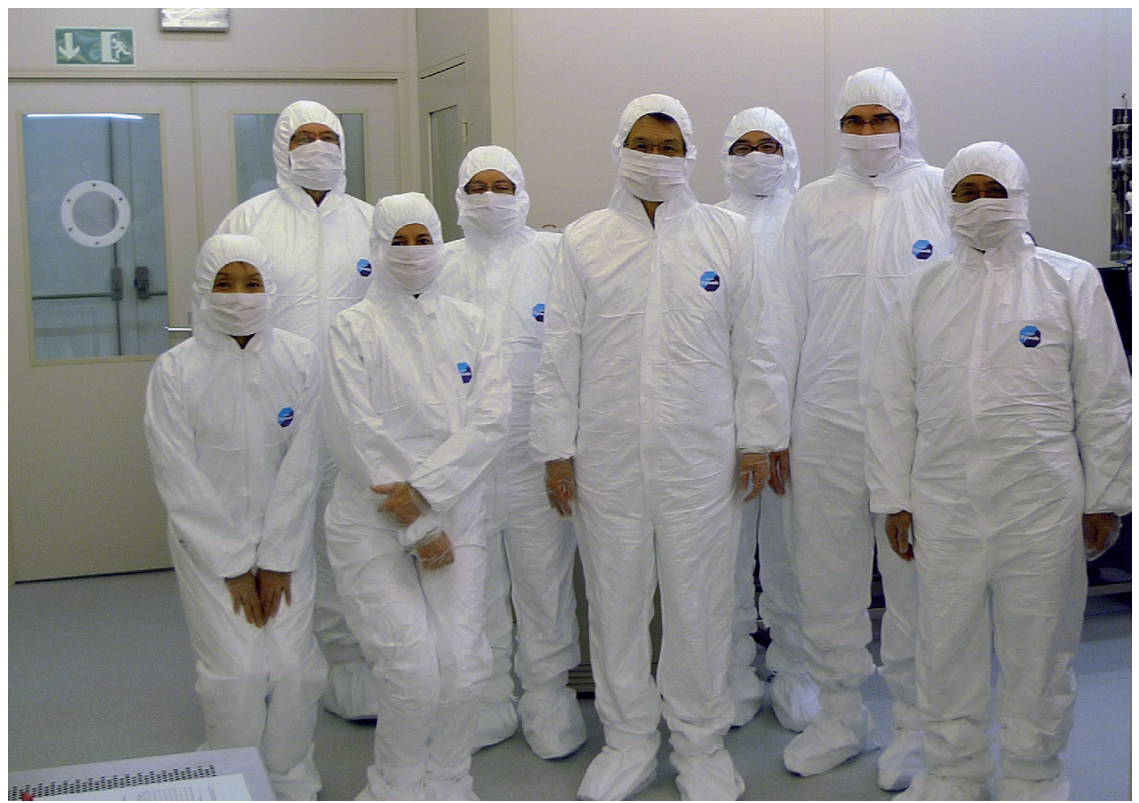

Fuente: Elaboración propia. 
del Ministerio de Ciencia e Innovación (MICINN). Por esta razón, en el resto de este artículo el acrónimo MICINN/MINECO para referirse a las dos entidades responsables de la ciencia y tecnología españolas durante el periodo $2008-2015$.

\section{Las convocatorias de proyectos conjuntos sobre nano- tecnología para retos energéticos y medioambientales}

El taller celebrado en Tokio en 2009 sirvió para analizar el potencial de la colaboración entre el MICINN/MINECO y la agencia japonesa JST, permitiendo que ese mismo año se firmase un acuerdo específico de colaboración para financiar proyectos conjuntos de investigación en la temática de la nanotecnología orientada a la resolución de los retos energéticos y medioambientales temas de interés estratégico para ambos países. Con este programa se esperaba obtener resultados científicos que permitieran un avance significativo en ciencia y tecnología y pudieran dar lugar a desarrollos con aplicaciones industriales y de utilidad social. Mediante este acuerdo, España se convirtió en uno de los 23 países con los que la agencia JST establecía relaciones dentro de su Programa Cooperativo de Investigación Internacional Estratégica (SIRCP) (SIRCP, 2015). Se debe mencionar que en el año 2010, el MICINN/MINECO aprobó el proyecto "Fomento de la cooperación con Rusia, Australia y Asia en el Área de la nanotecnologías (NANORAA)", cuya finalidad fue la de realizar contactos exploratorios y promocionar la nanotecnología española en regiones con las que históricamente existió un menor contacto científico.

\section{Temáticas de las convocatorias}

En el contexto del convenio entre JST y MICINN/MINECO antes mencionado, se estableció un esquema de financiación de proyectos conjuntos, basado en tres convocatorias en concurrencia competitiva, a las que se presentarían consorcios formados por grupos de investigación (tanto de entidades públicas como de empresas) de ambos países. Los proyectos realizarían propuestas para utilizar las nanotecnologías en la generación de nuevos materiales y dispositivos con el fin de potenciar las fuentes de energía renovable, optimizar el uso de las no renovables y fomentar los procesos de producción eficientes y sostenibles. Las temáticas principales en las que se agruparon los proyectos fueron:

a. Energía fotovoltaica.

b. Nanomateriales para química verde, eficiente y sostenible.

c. Nuevos materiales y dispositivos para generación, transformación y almacenamiento eficiente de energía.

d. Materiales para pilas de combustible, almacenamiento de hidrógeno y nuevas baterías. 


\section{Solicitudes recibidas y metodología de evaluación empleada}

Se realizaron 3 convocatorias conjuntas en los años 2009, 2010 y 2011, a las que se presentaron 61,36 , y 16 solicitudes, respectivamente (figura 4). Las solicitudes se presentaban por medios telemáticos simultáneamente tanto en MICINN/MINECO como en la agencia JST. En cada convocatoria se dispuso de un mes de plazo para el envío de las solicitudes. En total fueron aprobados un total 17 de proyectos: 8 en 2009, 4 en 2010 y 5 en 2011, lo cual demuestra que las convocatorias fueron muy competitivas, pues sólo el $15 \%$ de las solicitudes fueron financiadas. Como dato curioso, cabe mencionar que la convocatoria de 2009, con 61 solicitudes, supuso para la agencia JST el record histórico de participación en una convocatoria de sus programas de colaboración científica internacional. Este hecho revela el gran interés por establecer colaboraciones por parte de los grupos de investigación de ambos países.

En cada convocatoria las solicitudes recibidas fueron evaluadas inicialmente de forma separada por cada entidad convocante por los paneles de expertos configurados para la ocasión. Por lo general, cada proyecto recibió 2 o 3 evaluaciones por cada entidad convocante (MINCINN/MINECO y JST). En conjunto, más de medio centenar de expertos de los dos países han estado involucrados en la evaluación de las propuestas de las tres convocatorias. Una vez emitidos los informes de evaluación, pasaban a una segunda fase los proyectos en los que existía mayor consenso en cuanto a su buena calificación. La decisión final sobre los proyectos aprobados en cada convocatoria se

FIGURA 4. Proyectos presentados a las convocatorias de los años 2009, 2010 y 2011, dentro del convenio conjunto hispanojaponés sobre "Nanotecnología para los retos energéticos y medioambientales", indicando los proyectos aprobados y financiados (región más oscura) y no aprobados (región más clara).

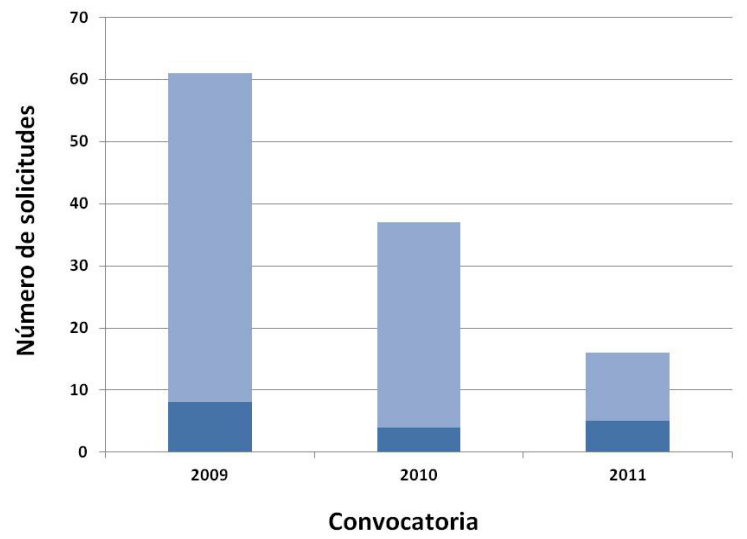

Fuente: Elaboración propia. 
llevó a cabo, para cada convocatoria, en una reunión conjunta de representantes de ambos paneles de expertos, que realizaba la priorización de las propuestas atendiendo a los criterios de calidad científica, originalidad, oportunidad, impacto en la economía de los dos países, capacidad de formación de nuevo personal investigador, etcétera.

Es importante mencionar que la puesta en marcha de este programa entre MICINN/MINECO y la agencia JST supuso para la parte española poner en marcha una metodología de trabajo que acortase el tiempo que habitualmente se requiere para la concesión de un proyecto desde la publicación de la convocatoria. Por lo común, la convocatoria se publicaba en mayo/ junio de cada año y la resolución de concesión se publicaba en octubre/noviembre del mismo año, lo que significaba acortar un 20-25\% el tiempo que se requería habitualmente.

\section{Relación de los proyectos aprobados}

De los 17 proyectos aprobados, uno, perteneciente a la convocatoria de 2010, fue cancelado durante su ejecución debido a problemas administrativos; así se desarrollaron completamente un total de 16 proyectos conjuntos entre el año 2010 y el año 2015.

La tabla 1 recoge el listado de los 16 proyectos aprobados y ejecutados, incluyendo los títulos de los proyectos, los investigadores principales, las entidades involucradas en su ejecución y las temáticas generales en las que se encuadraron. Se debe mencionar que en la convocatoria del año 2009, además de los 8 proyectos conjuntos financiados, el MICINN/MINECO financió 15 Acciones Especiales de Coordinación a otros tantos grupos españoles, con el fin de que éstos establecieran contacto con grupos japoneses. Estas acciones, únicamente dirigidas a los grupos españoles, no se tienen en cuenta en el presente estudio, aunque también sirvieron de manera indiscutible para incentivar la colaboración entre los grupos de ambos países.

Cada entidad convocante y financiadora (JST y MICINN/MINECO) concedió recursos económicos a los grupos de su país. Estos recursos se utilizaron para financiar, con bastante flexibilidad, tanto para cubrir la contratación de personal como los gastos de funcionamiento (pequeño equipamiento, material fungible, participación en congresos, viajes y estancias de investigadores, y la organización de talleres de trabajo y reuniones conjuntas). La tabla 2 recoge el número total de investigadores involucrados en los 16 proyectos aprobados. En total, 342 investigadores han participado en los proyectos, sin contar el número de investigadores que posteriormente fueron contratados con cargo a dichos proyectos. En un análisis de participación por sexos, hay que destacar que dos investigadoras españolas lideraron proyectos, mientras que por la parte japonesa todos los líderes de los proyectos fueron hombres. Además, el porcentaje de mujeres participantes en los grupos españoles fue superior al de los grupos japoneses, poniendo de manifiesto 
Mundo Nano | Artículos | Vol. 8, No. 15, julio-diciembre, 2015 | www.mundonano.unam.mx

TABLA 1. Proyectos aprobados y financiados en las convocatorias 2009, 2010 y 2011 dentro del convenio conjunto hispanojaponés sobre "Nanotecnología para los retos energéticos y medioambientales".

\begin{tabular}{|c|c|c|c|c|}
\hline $\begin{array}{l}\text { TIITULO DEL } \\
\text { PROYECTO }\end{array}$ & $\begin{array}{c}\text { INVESTIGADOR } \\
\text { PRINCIPAL ESPAÑOL } \\
\end{array}$ & $\begin{array}{c}\text { INVESTIGADOR } \\
\text { PRINCIPAL JAPONÉS } \\
\end{array}$ & $\begin{array}{c}\text { AÑO DE } \\
\text { CONVOCATORIA } \\
\end{array}$ & $\begin{array}{c}\text { ÁREA } \\
\text { TEMÁTICA (*) }\end{array}$ \\
\hline $\begin{array}{l}\text { Diseño, exploración y } \\
\text { fabricación de células } \\
\text { solares de alta } \\
\text { eficiencia de } \\
\text { conversión energética } \\
\text { basadas en nuevos } \\
\text { materiales }\end{array}$ & $\begin{array}{l}\text { Abderrazzak Douhal } \\
\text { (Instituto de Nano- } \\
\text { tecnología, Nano- } \\
\text { ciencia y Materiales } \\
\text { Moleculares - } \\
\text { INAMOL-, Universidad } \\
\text { de Castilla La Mancha) }\end{array}$ & $\begin{array}{l}\text { Shuji Hayase } \\
\text { (Kyushu Institute of } \\
\text { Technology) }\end{array}$ & 2009 & A \\
\hline $\begin{array}{l}\text { Células solares } \\
\text { nanodiseñadas de } \\
\text { bajo coste basadas en } \\
\text { nanocristales } \\
\text { semiconductores }\end{array}$ & $\begin{array}{l}\text { Juan Bisquert } \\
\text { Mascarell (Universitat } \\
\text { Jaume I) }\end{array}$ & $\begin{array}{l}\text { Taro Toyoda (The } \\
\text { University of Electro- } \\
\text { Communications) }\end{array}$ & 2009 & A \\
\hline $\begin{array}{l}\text { Estructuras de puntos } \\
\text { cuánticos de alta } \\
\text { densidad para células } \\
\text { solares de banda } \\
\text { intermedia }\end{array}$ & \begin{tabular}{|l|} 
Antonio Luque \\
(Instituto de Energía \\
Solar, Universidad \\
Politécnica de Madrid)
\end{tabular} & $\begin{array}{l}\text { Yoshitaka Okada } \\
\text { (University of Tokyo) }\end{array}$ & 2009 & A \\
\hline $\begin{array}{l}\text { Nuevos materiales } \\
\text { para electrolitos y } \\
\text { electrodos para } \\
\text { baterías de litio } \\
\text { sólidas de lámina } \\
\text { delgada }\end{array}$ & \begin{tabular}{|l|} 
Mario Aparicio \\
(Instituto de Cerámica \\
y Vidrio, Consejo \\
Superior de \\
Investigaciones \\
Científicas) \\
\end{tabular} & $\begin{array}{l}\text { Masahiro Tatsumisago } \\
\text { (Osaka Prefecture } \\
\text { University) }\end{array}$ & 2009 & D \\
\hline $\begin{array}{l}\text { Monolitos nano- } \\
\text { estructurados de } \\
\text { carbón para alma- } \\
\text { cenamiento y conver- } \\
\text { sión de metano }\end{array}$ & $\begin{array}{l}\text { Francisco Rodríguez- } \\
\text { Reinoso } \\
\text { (Universidad de } \\
\text { Alicante) }\end{array}$ & $\begin{array}{l}\text { Katsumi Kaneko } \\
\text { (Graduate School of } \\
\text { Science and } \\
\text { Technology, Chiba } \\
\text { University) } \\
\end{array}$ & 2009 & D \\
\hline $\begin{array}{l}\text { Nuevos catalizadores } \\
\text { de oro para la química } \\
\text { sostenible y una } \\
\text { conversión energética } \\
\text { eficiente }\end{array}$ & $\begin{array}{l}\text { Avelino Corma } \\
\text { (Instituto de Tecno- } \\
\text { logías Químicas, CSIC } \\
\text { - Universidad Politéc- } \\
\text { nica de Valencia) }\end{array}$ & $\begin{array}{l}\text { Mastake Haruta } \\
\text { (Tokyo Metropolitan } \\
\text { University) }\end{array}$ & 2009 & B \\
\hline $\begin{array}{l}\text { Nuevas zeolitas para } \\
\text { procesos de } \\
\text { separación de gases y } \\
\text { producción sostenible } \\
\text { de productos químicos }\end{array}$ & $\begin{array}{l}\text { Fernando Rey } \\
\text { (Instituto de Tecno- } \\
\text { logías Químicas, CSIC } \\
\text { - Universidad Politéc- } \\
\text { nica de Valencia) } \\
\end{array}$ & $\begin{array}{l}\text { Takashi Tatsumi } \\
\text { (Tokyo Institute of } \\
\text { Technology) }\end{array}$ & 2009 & B \\
\hline $\begin{array}{l}\text { Primeros pasos hacia } \\
\text { la integración de } \\
\text { arrays de nanohilos } \\
\text { en dispositivos } \\
\text { termoeléctricos útiles } \\
\text { para aplicaciones } \\
\text { energéticas }\end{array}$ & \begin{tabular}{|l|} 
Marisol Martín- \\
González (Instituto de \\
Microelectrónica de \\
Madrid, Consejo \\
Superior de \\
Investigaciones \\
Científicas)
\end{tabular} & $\begin{array}{l}\text { Yoshikazu Shinohara } \\
\text { (National Institute for } \\
\text { Materials Science - } \\
\text { NIMS) }\end{array}$ & 2009 & C \\
\hline
\end{tabular}


TABLA 1. (Continuación).

\begin{tabular}{|c|c|c|c|c|}
\hline $\begin{array}{l}\text { TÍTULO DEL } \\
\text { PROYECTO }\end{array}$ & $\begin{array}{c}\text { INVESTIGADOR } \\
\text { PRINCIPAL ESPAÑOL }\end{array}$ & $\begin{array}{c}\text { INVESTIGADOR } \\
\text { PRINCIPAL JAPONÉS }\end{array}$ & $\begin{array}{c}\text { AÑO DE } \\
\text { CONVOCATORIA } \\
\end{array}$ & $\begin{array}{c}\text { ÁREA } \\
\text { TEMÁTICA (*) }\end{array}$ \\
\hline $\begin{array}{l}\text { Endofulerenos para } \\
\text { dispostivos } \\
\text { fotovoltaicos }\end{array}$ & $\begin{array}{l}\text { Nazario Martín } \\
\text { (Universidad } \\
\text { Complutense de } \\
\text { Madrid) }\end{array}$ & $\begin{array}{l}\text { Takeshi Akasaka } \\
\text { (University of } \\
\text { Tsukuba) }\end{array}$ & 2010 & A \\
\hline $\begin{array}{l}\text { Nanoplataformas } \\
\text { multifuncionalesde } \\
\text { alta sensitividad para } \\
\text { control y purificación } \\
\text { de agua }\end{array}$ & $\begin{array}{l}\text { Arben Merkoçi } \\
\text { (Fundación Privada } \\
\text { Institut Català de } \\
\text { Nanotecnologia) }\end{array}$ & $\begin{array}{l}\text { Akira Fujishima } \\
\text { (Tokyo University of } \\
\text { Science) }\end{array}$ & 2010 & B \\
\hline $\begin{array}{l}\text { Nuevos materials de } \\
\text { tipo perovskita como } \\
\text { componentes de } \\
\text { dispositivos } \\
\text { electroquímicos para } \\
\text { generación de energía }\end{array}$ & $\begin{array}{l}\text { Susana García Martín } \\
\text { (Universidad } \\
\text { Complutense de } \\
\text { Madrid) }\end{array}$ & $\begin{array}{l}\text { Nobuyuki Imanishi } \\
\text { (Mie University) }\end{array}$ & 2010 & D \\
\hline $\begin{array}{l}\text { Core-Shell LEDs } \\
\text { basados en sistemas } \\
\text { de nanocolumnas de } \\
\text { ZnMgO con altas } \\
\text { eficiencias } \\
\text { electrónicas }\end{array}$ & $\begin{array}{l}\text { Adrián Hierro } \\
\text { (Universidad } \\
\text { Politécnica de Madrid) }\end{array}$ & $\begin{array}{l}\text { Atsushi Nakamura } \\
\text { (Research Institute of } \\
\text { Electronics, Shizuoka } \\
\text { University) }\end{array}$ & 2010 & C \\
\hline $\begin{array}{l}\text { Nuevos materiales, } \\
\text { materiales } \\
\text { compuestos de } \\
\text { nanocristales- } \\
\text { polímeros para células } \\
\text { solares inorgánicas de } \\
\text { bajo coste y alta } \\
\text { eficiencia procesadas } \\
\text { en disolución }\end{array}$ & \begin{tabular}{|l} 
Gerasimos \\
Konstantatos \\
(Instituto de Ciencias \\
Fotónicas -ICFO)
\end{tabular} & $\begin{array}{l}\text { Keisuke Tajima } \\
\text { (The University of } \\
\text { Tokyo) }\end{array}$ & 2011 & A \\
\hline $\begin{array}{l}\text { Caracterización y } \\
\text { desarrollo de } \\
\text { materiales avanzados } \\
\text { para el desarrollo de } \\
\text { nuevas baterías de } \\
\text { litio }\end{array}$ & $\begin{array}{l}\text { Manuel Perlado } \\
\text { (Instituto de Fusión } \\
\text { Nuclear, Universidad } \\
\text { Politécnica de Madrid) }\end{array}$ & $\begin{array}{l}\text { Yoshiaki Kato } \\
\text { (The Graduate School } \\
\text { for the Creation of } \\
\text { New Photonics } \\
\text { Industries) }\end{array}$ & 2011 & D \\
\hline $\begin{array}{l}\text { Nuevos materiales } \\
\text { carbonosos con } \\
\text { propiedades únicas } \\
\text { para superconden- } \\
\text { sadores híbridos } \\
\text { asimétricos }\end{array}$ & $\begin{array}{l}\text { Diego Cazorla Amorós } \\
\text { (Universidad de } \\
\text { Alicante) }\end{array}$ & $\begin{array}{l}\text { Takashi Kyotani } \\
\text { (Institute of } \\
\text { Multidisciplinary } \\
\text { Research for } \\
\text { Advanced Materials } \\
\text { Tohoku University) }\end{array}$ & 2011 & C \\
\hline
\end{tabular}


TABLA 1. (Continuación).

\begin{tabular}{|c|c|c|c|c|}
\hline $\begin{array}{l}\text { TÍTULO DEL } \\
\text { PROYECTO }\end{array}$ & $\begin{array}{c}\text { INVESTIGADOR } \\
\text { PRINCIPAL ESPAÑOL }\end{array}$ & $\begin{array}{l}\text { INVESTIGADOR } \\
\text { PRINCIPAL JAPONÉS }\end{array}$ & $\begin{array}{c}\text { AÑO DE } \\
\text { CONVOCATORIA }\end{array}$ & $\begin{array}{c}\text { ÁREA } \\
\text { TEMÁTICA (*) }\end{array}$ \\
\hline $\begin{array}{l}\text { Desarrollo de } \\
\text { dispositivos basados } \\
\text { en películas delgadas } \\
\text { termoeléctricas } \\
\text { usando } \\
\text { nanoestructuras } \\
\text { magnéticas para } \\
\text { generación } \\
\text { termoeléctrica }\end{array}$ & $\begin{array}{l}\text { Manuel Ricardo Ibarra } \\
\text { García } \\
\text { (Instituto de } \\
\text { Nanociencia de } \\
\text { Aragón, Universidad } \\
\text { de Zaragoza) }\end{array}$ & $\begin{array}{l}\text { Sadamichi Maekawa } \\
\text { (Advanced Science } \\
\text { Research Center, } \\
\text { Japan Atomic Energy } \\
\text { Agency) }\end{array}$ & 2011 & C \\
\hline \multicolumn{5}{|c|}{$\begin{array}{l}\text { * Claves de las áreas temáticas: } \\
\text { A. Energía fotovoltaica. } \\
\text { B. Nanomateriales para química verde, eficiente y sostenible. } \\
\text { C. Nuevos materiales y dispositivos para generación, transformación y almacenamiento eficientes } \\
\text { de energía. } \\
\text { D. Materiales para pilas de combustible, almacenamiento de hidrógeno y nuevas baterías. }\end{array}$} \\
\hline
\end{tabular}

Fuente: Elaboración propia.

TABLA 2. Número de investigadores participantes en los 16 proyectos aprobados y financiados en las tres convocatorias dentro del convenio conjunto hispanojaponés sobre "Nanotecnología para los retos energéticos y medioambientales".

\begin{tabular}{|l|c|c|c|c|}
\hline & $\begin{array}{c}\text { INVESTIGADORES } \\
\text { DE PLANTILLA }\end{array}$ & $\begin{array}{c}\text { DOCTORES CONTRATADOS } \\
\text { (POSTDOCS) }\end{array}$ & $\begin{array}{c}\text { INVESTIGADORES } \\
\text { EN FORMACIÓN }\end{array}$ & TOTAL \\
\hline Grupos japoneses & 84 & 27 & 81 & 192 \\
\hline Grupos españoles & 61 & 47 & 42 & 150 \\
\hline Total & 145 & 74 & 123 & 342 \\
\hline
\end{tabular}

Fuente: Elaboración propia.

uno de los problemas de la ciencia japonesa: la escasa integración de mujeres en los grupos de investigación.

Otro aspecto que se debe destacar es la inversión total realizada por las entidades convocantes. La tabla 3 recoge las cantidades que las dos entidades, JST y MICINN/MINECO, destinaron para financiar los 16 proyectos. La inversión conjunta ha sido de 6,2 M€, con una financiación promedio de $386 \mathrm{k} €$ por proyecto, cantidad bastante elevada para este tipo de convocatorias conjuntas de colaboración internacional. El esfuerzo estuvo bien repartido entre las dos entidades convocantes, correspondiendo el 52,5\% al MICINN/MINECO y el $47,5 \%$ a la agencia JST. Este dato demuestra que el convenio de colaboración fue planteado en términos de igualdad entre dos naciones que poseen un importante peso en el contexto mundial. 
TABLA 3. Inversión realizada por las entidades convocantes (JST y MICINN/MINECO) en los 16 proyectos aprobados y financiados en las tres convocatorias dentro del convenio conjunto hispanojaponés sobre "Nanotecnología para los retos energéticos y medioambientales".

\begin{tabular}{|l|c|c|c|}
\hline & $\begin{array}{c}\text { FINANCIACIÓN DE JST } \\
\text { PARA GRUPOS } \\
\text { JAPONESES (YEN) }\end{array}$ & $\begin{array}{c}\text { FINANCIACIÓN DE MICINN / } \\
\text { MINECO PARA GRUPOS } \\
\text { ESPAÑOLES (EURO) }\end{array}$ & $\begin{array}{c}\text { FINANCIACIÓN CONJUNTA TOTAL } \\
\text { (JST + MICINN/MINECO) (EURO)* }\end{array}$ \\
\hline $\begin{array}{l}\text { Total de las tres } \\
\text { convocatorias }\end{array}$ & $363,364,750.00$ & $3,248,484.00$ & $6,178,817.93$ \\
\hline $\begin{array}{l}\text { Promedio por } \\
\text { proyecto }\end{array}$ & $22,710,296.88$ & $203,030.25$ & $386,176.12$ \\
\hline
\end{tabular}

(*) Las cantidades en yenes se han convertido en euros teniendo en cuenta el cambio promedio de divisa del año de la concesión.

Fuente: Elaboración propia.

\section{Metodología empleada para el seguimiento de los proyectos aprobados}

Además de todos los procesos de gestión necesarios para convocar y resolver las convocatorias conjuntas, se estableció un mecanismo de seguimiento de los proyectos a lo largo de su desarrollo. De esta forma, al concluir el segundo año de ejecución del proyecto, se valoró por parte de un comité de expertos designado por el MICINN/MINECO y la agencia JST, la evolución y resultados de cada proyecto. Aprovechando la presencia de los investigadores españoles y japoneses, las entidades colaboradoras organizaron talleres o reuniones científicas abiertos a la comunidad científica de los dos países. De esta manera, se celebraron las siguientes jornadas de seguimiento y talleres científicos:

- Jornada de seguimiento conjunto de los proyectos financiados de la convocatoria 2009 y celebración del 3th Workshop "Nanociencias y Nuevos Materiales" (Toledo, España, 2011).

- Jornada de seguimiento conjunto de los proyectos financiados de la convocatoria 2010 y celebración del 4th Workshop "Nanociencias y Nuevos Materiales" (Tsukuba, Japón, 2013).

- Jornada de seguimiento conjunto de los proyectos financiados de la convocatoria 2011 y celebración de la sesión conjunta JST-MINECO dentro del congreso Trends in Nanotechnology Japan 2014 (Tokio, Japón, 2014). Este congreso formó parte de las actividades programadas dentro del Año Dual España-Japón, una iniciativa conjunta hispanojaponesa orientada a intensificar y abrir nuevos horizontes a unas relaciones bilaterales centenarias (celebrada entre octubre de 2013 y septiembre de 2014). 
TABLA 4. Promedio de las valoraciones efectuadas en las jornadas de seguimiento de los 16 proyectos aprobados y financiados en las tres convocatorias dentro del convenio conjunto hispanojaponés sobre "Nanotecnología para los retos energéticos y medioambientales".

\begin{tabular}{|l|c|}
\hline ASPECTO VALORADO & VALORACIÓN (0-5) \\
\hline Seguimiento del plan de investigación propuesto & 4.3 \\
\hline Resultados alcanzados en el segundo años de proyecto & 3.9 \\
\hline Ejecución del programa de intercambio de investigadores & 3.8 \\
\hline Conjunto del proyecto & 3.9 \\
\hline
\end{tabular}

Fuente: Elaboración propia.

Se debe mencionar que la evaluación intermedia de los proyectos es una aportación realizada desde MICINN/MINECO ya que no era práctica habitual en JST la realización de un seguimiento de este tipo. La tabla 4 recoge, sobre una escala de 0 a 5 , las valoraciones recibidas por los proyectos en dichas jornadas de seguimiento, que, como puede apreciarse, han sido bastante positivas, indicando que los proyectos se fueron desarrollando de acuerdo al plan propuesto y que los resultados han sido notables.

Una vez concluidos los proyectos, las dos entidades MICINN/MINECO y JST recibieron y analizaron informes finales donde se detallaban las actividades realizadas y se justificaron convenientemente los recursos obtenidos. Dichos informes finales, de carácter conjunto, han sido analizados en las dos entidades financiadoras y los resultados obtenidos se han calificado de muy satisfactorios. Estos informes finales también han servido para realizar el análisis que se muestra en la siguiente sección.

\section{Análisis de los resultados de las convocatorias conjuntas}

Tal y como se recoge en la tabla 1 , cinco proyectos concedidos han estado relacionados con la energía fotovoltaica, proponiendo la mejora de células solares basadas en semiconductores mediante nanoestructuras o materiales nanocompuestos, así como la propuesta de nuevos materiales orgánicos para su uso en generación fotovoltaica. Por otro lado, se ejecutaron otros cinco proyectos relacionados con la transformación y almacenamiento eficientes de energía basados en materiales avanzados, destacando el desarrollo de dispositivos termoeléctricos, sistemas de diodos de emisión láser (LED) de alta eficiencia y nuevos supercondensadores basados en carbono. En relación con la mejora de baterías o el uso del hidrógeno se aprobaron diferentes proyectos destacando proyectos innovadores en los que se proponen sistemas de lámina delgada para electrodos de baterías o complejos materiales de tipo perovskita para el transporte iónico. Finalmente, una serie de proyectos estaban más relacionados con la mejora de la eficiencia de procesos físicoquímicos para la purificación de agua, la separación de gases, o la 
producción de diferentes sustancias de interés industrial. Como ya se ha puesto de manifiesto por muchos expertos (Gómez-Romero, 2007), el impacto de la nanotecnología en el ámbito de la energía y la sostenibilidad es muy importante, realizando imaginativas propuestas, algunas de las cuales podrán llevarse a medio plazo al mercado. Por lo tanto, un primer éxito de la convocatoria es la completitud de los temas abordados, permitiendo poner sobre la mesa diferentes aproximaciones para resolver diferentes problemas.

Los resultados de los proyectos de investigación son cuantificables de diferentes maneras, aunque lo más habitual es utilizar un conjunto de indicadores. En el caso de la convocatoria conjunta JST-MINECO, no se establecieron a priori indicadores de objetivos a alcanzar en cada proyecto, debido a que en el ámbito de la ciencia más básica los resultados obtenidos suelen diferir de los planeados, dado que la propia investigación cierra caminos inicialmente previstos pero abre otros no esperados. Además, en temas frontera, como los relacionados con la nanociencia y la nanotecnología, la incertidumbre es mayor que en temas de investigación más consolidados. Por lo tanto, es difícil definir una serie de indicadores a priori que permitan medir el grado de cumplimiento o éxito del conjunto de la convocatoria. Por tanto, los indicadores disponibles son aquellos obtenidos a posteriori, una vez finalizados los proyectos.

En la tabla 5 se recogen algunos de los indicadores de producción científicotécnica que se han conseguido por los 16 proyectos financiados por las dos entidades convocantes. Los resultados mostrados se obtuvieron a través de una encuesta realizada entre los grupos de investigación y usando los informes enviados por los grupos a sus respectivas entidades financiadoras. La tabla 5 muestra tanto la producción total de los grupos, como aquella que corresponde exclusivamente a la producción conjunta derivada de los proyectos financiados en las tres convocatorias. Debemos destacar que los datos recogidos no son homogéneos pues los proyectos de la primera convocatoria han tenido mayor tiempo para poder publicar resultados una vez concluida formalmente su financiación. Los proyectos finalizados más recientemente todavía tienen potencial para dar lugar a más publicaciones, por lo que los datos no son definitivos, pero sí dan una idea aproximada de la producción conjunta del programa colaborativo.

En conjunto se observa que los proyectos han contribuido a fomentar la interacción entre los grupos y que esta interacción se ha plasmado en publicaciones en revistas internacionales, libros, y patentes. Algunos de esos resultados se han plasmado en obras conjuntas sin excluir la publicación de obras con autoría de miembros pertenecientes a un solo grupo de investigación. Los 62 artículos realizados en colaboración directa entre los grupos españoles y los japoneses han sido publicados en revistas de diferente impacto, pudiéndose destacar que 20 de las mismas lo han sido en revistas con índice de impacto superior a 6 . La tabla 6 recoge una selección de las publicaciones realizadas en el marco de los 16 proyectos financiados. En cuanto a las 
Mundo Nano | Artículos | Vol. 8, No. 15, julio-diciembre, 2015 | www.mundonano.unam.mx

TABLA 5. Principales indicadores de resultados obtenidos por los 16 proyectos aprobados en las tres convocatorias dentro del convenio conjunto hispanojaponés sobre "Nanotecnología para los retos energéticos y medioambientales".

\begin{tabular}{|l|c|c|c|}
\hline & $\begin{array}{c}\text { PRODUCCIÓN CONJUNTA } \\
\text { (CON AUTORES DE LOS } \\
\text { GRUPOS ESPAÑOL Y } \\
\text { JAPONÉS) }\end{array}$ & $\begin{array}{c}\text { PRODUCCIÓN TOTAL } \\
\text { OBTENIDA POR LOS } \\
\text { GRUPOS EN EL } \\
\text { PERIODO ANALIZADO }\end{array}$ & $\begin{array}{c}\text { PRODUCCIÓN } \\
\text { CONJUNTA SOBRE } \\
\text { LA PRODUCCIÓN } \\
\text { TOTAL }\end{array}$ \\
\hline $\begin{array}{l}\text { Número de publicaciones en } \\
\text { revistas internacionales }\end{array}$ & 62 & 465 & 13.33 \\
\hline $\begin{array}{l}\text { Número de publicaciones en } \\
\text { revistas internacionales con un } \\
\text { factor de impacto mayor que 6 }\end{array}$ & 20 & 129 & 15.50 \\
\hline Número de capítulos de libro & 3 & 45 & 6.67 \\
\hline Número de libros completos & 0 & 7 & 0.00 \\
\hline $\begin{array}{l}\text { Número de comunicaciones } \\
\text { orales y conferencias invitadas } \\
\text { presentadas en congresos y } \\
\text { talleres internacionales }\end{array}$ & 66 & 351 & 18.80 \\
\hline $\begin{array}{l}\text { Número de patentes } \\
\text { internacionales registradas }\end{array}$ & 1 & 6 & 16.67 \\
\hline
\end{tabular}

Fuente: Elaboración propia.

actividades más cercanas a la transferencia de tecnología, es cierto que el número de patentes conjuntas es muy inferior al de artículos o capítulos de libro debido a que las patentes son resultado de un proceso más largo de maduración y colaboración. Por otro lado, en algunos casos se detectó cierta reticencia a trabajar en patentes debido a las implicaciones económicas que éstas tienen para las instituciones.

Además de los anteriores indicadores, hay que mostrar que los resultados de la colaboración también han servido para incentivar intercambio de investigadores y la organización de eventos de manera conjunta, ayudando a un mejor conocimiento mutuo de las dinámicas de trabajo propias de cada país. En total se han realizado 117 reuniones de trabajo conjuntas (más de 7 por proyecto) entre los grupos españoles y japoneses para coordinar las actividades de investigación. Un total de 104 investigadores japoneses han realizado visitas a los grupos españoles (con una duración promedio de 10 días) y un total de 63 investigadores españoles han visitado a sus socios japoneses (con una duración promedio de 30 días). En conjunto, se han realizado 167 intercambios, lo que representa más de 10 por proyecto financiado.

En cuanto al efecto de los proyectos sobre las capacidades de los grupos, se debe destacar que también ha existido un impacto directo sobre la formación del personal participante en los proyectos de investigación. De esta forma, se han defendido 44 tesis de máster (32 en Japón y 12 en España) y 35 tesis de doctorado (13 en Japón y 22 en España) en temáticas 
TABLA 6. Selección de artículos publicados en revistas internacionales como resultado de los proyectos financiados en las tres convocatorias dentro del convenio conjunto hispanojaponés sobre "Nanotecnología para los retos energéticos y medioambientales".

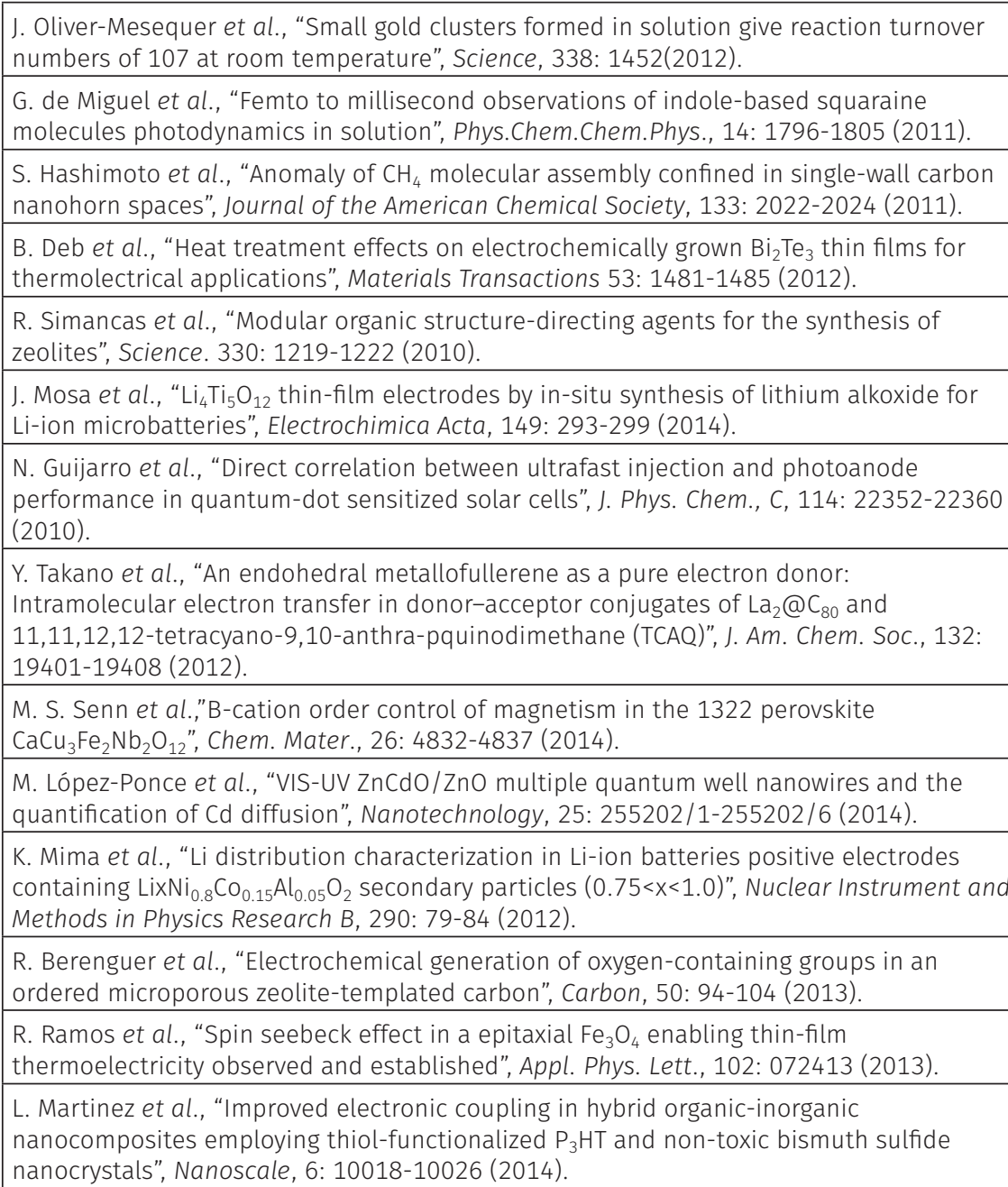

Fuente: Elaboración propia.

relacionadas con las de los proyectos de investigación. De hecho, una parte significativa de las estancias antes mencionadas corresponde a intercambios entre jóvenes investigadores en formación. Se debe mencionar que algunos de los investigadores españoles que han alcanzado el grado de doctor durante el desarrollo del proyecto han mostrado interés en incorporarse a grupos japoneses para realizar una estancia postdoctoral de larga duración. 
Otro indicador que puede ser útil para revelar el éxito del programa bilateral es el número de nuevas convocatorias internacionales a las que los grupos españoles y japoneses han enviado de manera conjunta solicitudes de proyectos con el fin de seguir manteniendo la colaboración alcanzada. En total se han presentado 4 proyectos a convocatorias internacionales de otras entidades (fundamentalmente de la Unión Europea).

Para finalizar esta sección se debe mencionar que los proyectos financiados han tenido cierta visibilidad en los medios, tanto españoles como japoneses, y se contabilizan un total de 35 artículos de divulgación, entrevistas o apariciones en los medios de comunicación, en donde aparecen investigadores de los grupos de investigación participantes en los proyectos de investigación.

Un análisis conjunto de los resultados de todo el programa ha sido realizado, con detalle y profundidad, por las dos entidades JST y MICINN/MINECO, poniendo sobre la mesa los aspectos positivos de la colaboración, así como otros aspectos que pueden mejorarse de cara a futuras rediciones de este esquema conjunto de financiación de proyectos. Dicho análisis ha dado lugar a este artículo así como a un folleto conjunto publicado entre ambas entidades (figura 5). Dicho folleto da cuenta, de forma resumida, de los principales resultados obtenidos por los 16 proyectos de investigación financiados. La presentación oficial de los resultados de todas las actuaciones de

FIGURA 5. Portada del folleto realizado conjuntamente por el MICINN/MINECO y la agencia JST, que recoge los resultados más importantes obtenidos por los proyectos realizados en el marco del convenio conjunto hispanojaponés sobre "Nanotecnología para los retos energéticos y medioambientales".

\section{Nanotechnologies and new materials for environmental challenges and sustainable development}

A collaborative experience between Japan and Spain 2009-2015
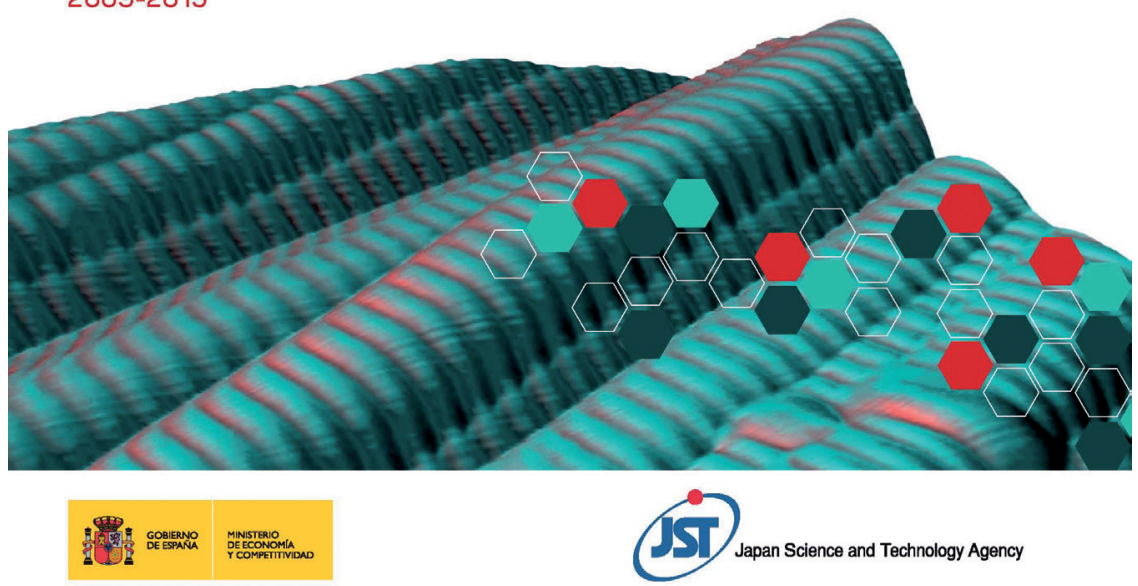
cooperación, y del folleto antes mencionado, tuvo lugar el 23 de octubre de 2015, en un acto celebrado en la Embajada de España en Japón, en el que participaron representantes políticos y científicos de ambos países, poniendo un broche de oro a todo un programa extendido durante casi 7 años desde la firma, en 2009, del Memorando de Entendimiento (MoU) entre el Ministerio de Ciencia e Innovación (MICINN) y la Agencia Japonesa de Ciencia y Tecnología (JST).

\section{Conclusiones y futuro}

La puesta en marcha de un programa de colaboración hispanojaponés en el ámbito de la aplicación de la nanotecnología para resolver retos energéticos y medioambientales ha sido una iniciativa desarrollada dentro de un contexto estratégico mucho más ambicioso de acercamiento mutuo entre España y Japón, países que han iniciado colaboraciones en diferentes temáticas cientificotécnicas. Este programa se ha basado en dos pilares: la financiación de proyectos conjuntos mediante tres convocatorias y la celebración de talleres científicos de alto nivel. En conjunto, los resultados obtenidos se pueden considerar satisfactorios por el número de publicaciones y de contribuciones a congresos. Además, se han producido un número elevado de estancias de investigadores españoles en Japón y de investigadores japoneses en España, lo que permite no sólo un intercambio de conocimientos científicos, sino sobre las dinámicas de trabajo que se emplean en otros países. Podemos mencionar que se han detectado ciertas dificultades en los temas de transferencia de tecnología, como el registro de patentes o la proyección industrial de los resultados. En conjunto, los proyectos han servido para consolidar relaciones ya existentes entre grupos españoles y japoneses, mientras en otros casos se han creado otras conexiones que seguramente requieran una mayor maduración. En el caso de los grupos españoles, los proyectos han permitido que investigadores españoles puedan realizar en Japón una estancia postdoctoral de larga duración en un grupo que va a mantener contactos con España, de forma que no se pierda la vinculación con el sistema de I+D+I nacional.

A su vez, el programa ha servido para realizar una puesta en común de metodologías en la gestión de la I+D+I, incluyendo el diseño de convocatorias, así como su seguimiento y evaluación. Estos resultados, que pasan a formar parte del capital intangible de las organizaciones, son casi tan importantes como los resultados científicos, pues permiten extrapolar las experiencias al diseño de futuras actuaciones. En este sentido, algunos aspectos que merece la pena destacar son:

- Se ha realizado una puesta en común de las metodologías de convocatorias y evaluación de proyectos. En concreto, se han realizado evaluaciones conjuntas mediante comisiones evaluadoras mixtas que inicialmente poseían mecánicas internas diferentes. 
- Se han debido acompasar ritmos diferentes a la hora de resolver convocatorias.

- Se han puesto en marcha jornadas de seguimiento intermedio de proyectos, que en el caso de la agencia japonesa no era una práctica habitual.

- Se ha sabido superar las dificultades surgidas por el hecho de que España y Japón poseen diferentes fechas de inicio para el año fiscal.

La figura 6 muestra la metodología empleada a lo largo del programa para realizar la gestión del mismo, resumiendo las principales características descritas en la anterior sección.

FIGURA 6. Representación esquemática de la metodología empleada por el MICINN/MINECO y la agencia JST para la puesta en marcha del convenio de colaboración y las convocatorias asociadas en el ámbito de la "Nanotecnología para los retos energéticos y medioambientales".

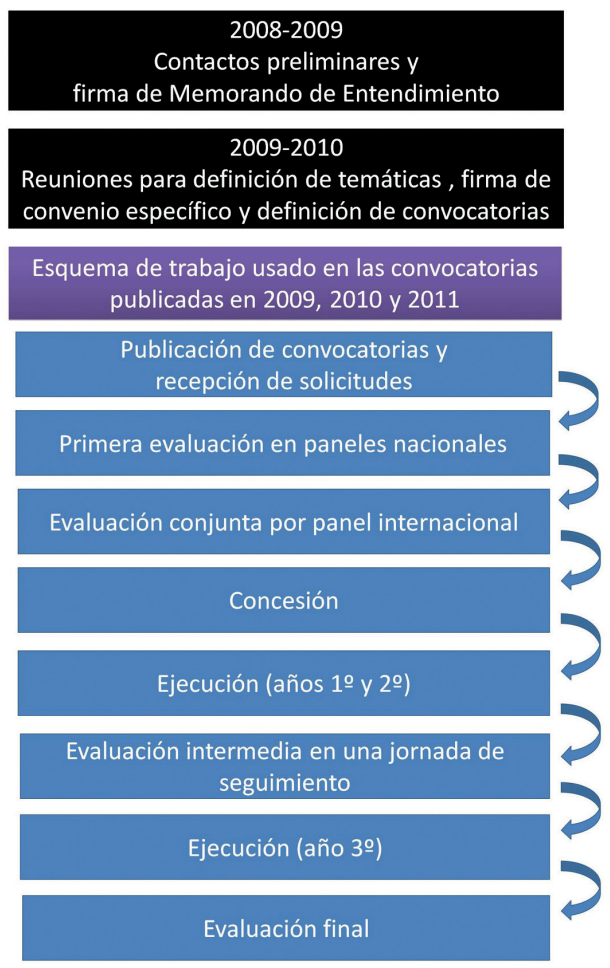

\section{5}

Evaluación final de todo el programa de colaboración y presentación conjunta de los resultados obtenidos por todos los proyectos

Fuente: Elaboración propia. 
Una vez terminado el programa conjunto, con sus éxitos y aprendizajes, creemos importante mantener una colaboración estable, aunque las circunstancias actuales en los dos países no son las más favorables para repetir un programa de las mismas dimensiones. No obstante, es necesario proponer otras actuaciones más focalizadas, más orientadas a resultados concretos en temas concretos. En este sentido, una buena temática relacionada con la nanotecnología, de interés para ambos países, y con un elevado impacto es la nanomedicina. Concretamente, en el área de nanomedicina, España cuenta con destacados grupos de investigación a nivel internacional agrupados en torno a la Plataforma Española de Nanomedicina (NanoMedSpain, 2015) y al Centro de Investigación Biomédica en Red en Bioingeniería, Biomateriales y Nanomedicina (CIBER-BBN, 2015) dependiente del Instituto de Salud Carlos III (ISCIII, 2015), el principal Organismo Público de Investigación (OPI), que financia, gestiona y ejecuta la investigación biomédica en España. Además, se debe mantener la presencia de la nanotecnología española en Japón a través del Pabellón Español en la Feria Nanotech Tokyo. A medio plazo, cuando el contexto económico sea más favorable, el mantenimiento de relaciones permitirá relanzar programas mucho más ambiciosos que permitan alcanzar éxito a partir de las experiencias acumuladas en estos últimos años.

\section{Referencias}

AIST (2015). National Institute of Advanced Industrial Science and Technology. <http://www.aist.go.jp/index_en.html>.

CDTI (2015). Centro para el Desarrollo Tecnológico Industrial. <https://www.cdti. es/>.

CIEMAT (2015). Centro de Investigaciones Energéticas, Medioambientales y Tecnológicas. <http://www.ciemat.es/>.

CSIC (2015). <http://www.csic.es >.

Delgado, G. C. (2009). Economía política de la nanotecnología. Mundo Nano Revista Interdisciplinaria en Nanociencia y Nanotecnología, 1(1): 87-94.

Esteban, Mario (2014). Dinamizando las relaciones hispano-japonesas: propuestas de la sociedad civil. Real Instituto Elcano, Madrid. Accesible en <http://foroespañajapon.es//wp-content/uploads/2014/09/Informe-Elcano-definitivo.pdf $>$.

Foro España Japón (2015). <http://www.spainjapanfoundation.com/>.

Gómez-Romero, Pedro (2007). Un planeta en busca de energía. Madrid: Editorial Síntesis.

ICEX (2015). ICEX España Exportación e Inversiones. <http://www.icex.es>.

JST (2015). Japan Science and Technology Agency. <http://www.jst.go.jp/EN/>.

JST-MICINN (2009). <http://www.jst.go.jp/sicp/ws2009_sp1st.html>.

MINECO (2015). Ministerio de Economía y Competitividad. <http://www.mineco. gob.es/portal/site/mineco/>.

NanoSpain (2015). Red Española de Nanotecnología. <http://www.nanospain.org>. 
Nanotech Tokyo (2015). International Nanotechnology Exhibition \& Conference. <http://www.nanotechexpo.jp>.

NEDO (2015). New Energy and Industrial Technology Development Organization. <http://www.nedo.go.jp/english/index.html>.

NIFS (2015). National Institute for Fusion Science. <http://www.nifs.ac.jp/en/index.html>.

NIMS (2015). National Institute for Materials Science. <http://www.nims.go.jp/ eng/>.

Noya, Javier (2004). La imagen de España en Japón. Madrid: Real Instituto Elcano. Accesible en <http://www.realinstitutoelcano.org/publicaciones/libros/librojapon.pdf>.

Phantoms (2015). Fundación Phantoms. <http://www.phantomsnet.net>.

Serena, P.A. (2009). La implantación de la nanotecnología en España: muchas luces y alguna sombra. Mundo Nano Revista Interdisciplinaria en Nanociencia y Nanotecnología, 2(2): 74-90. En: <http://www.mundonano.unam.mx/pdfs/mundonano3.pdf $>$.

SIRCP (2015). JST Strategic International Research Cooperative Program, <http:// www.jst.go.jp/inter/english/sicp/index.html>.

NanoMedSpain (2015). Plataforma Española de Nanomedicina. <http://www.nanomedspain.net/>.

CIBER-BBN (2015). Centro de Investigación Biomédica en Red en Bioingeniería, Biomateriales y Nanomedicina. <http://www.ciber-bbn.es/>.

ISCIII (2015). Instituto de Salud Carlos III. 〈http://www.isciii.es >.

NFI (2015). Naka Fusion Institute. <http://www.naka.jaea.go.jp/english/>.

ITER (2015). International Thermonuclear Experimental Reactor. <https://www. iter.org/>.

MEXT (2015). Ministry of Education, Culture, Sports, Science and Technology (MEXT). <http://www.mext.go.jp/english/a01.htm>. 